\title{
A Case of Distal Pancreatectomy for Pancreatic Metastasis of Thymic Atypical Carcinoid
}

\author{
Masashi Inoue $^{1, *}$, Masahiro Tanemura ${ }^{2}$, Toshimitsu Irei ${ }^{1}$, Yuyo Maeda ${ }^{1}$, Megumi Yamaguchi ${ }^{1}$, Tatsuya Miyamoto ${ }^{1}$, \\ Shingo Seo' ${ }^{1}$, Toshihiro Misumi ${ }^{1}$, Wataru Shimizu ${ }^{1}$, Takahisa Suzuki ${ }^{1}$, Takashi Onoe ${ }^{1}$, \\ Takeshi Sudo ${ }^{1}$, Yosuke Shimizu ${ }^{1}$, Takao Hinoi ${ }^{1}$, Hirotaka Tashiro ${ }^{1}$ \\ ${ }^{1}$ Department of surgery, Kure Medical Center Chugoku Cancer Center, Kure, Japan \\ ${ }^{2}$ Department of Surgery, Osaka police Hospital, Osaka, Japan \\ *Corresponding author: inoue_masashi@hiro-hosp.jp
}

\begin{abstract}
Background: Thymic atypical carcinoid(AC) tends to have a more aggressive clinical course than typical carcinoid(TC). About 20\%-30\% of patients with thymic AC experience local recurrence or distant metastasis. Pancreatic metastasis of thymic AC is extremely infrequent. We experienced a case of pancreatic metastasis of thymic atypical carcinoid, in which a distal pancreatectomy was performed. Case presentation: A 50-year-old man underwent thoracoscopic resection of a mediastinal tumor in June 2009. The diagnosis was thymic AC. He also underwent an extended thymectomy in October 2011, thoracoscopic resection in October 2012, and tumor resection of the chest wall in March 2013 for recurrences. During follow up in April 2014, computed tomography (CT) revealed lymph node swelling on the ventral side of the brachiocephalic vein, around the left gastric vein, and on the left side of the celiac artery. Dynamic CT revealed a pancreatic tumor $10 \mathrm{~mm}$ in diameter with low enhancement. EUS-guided fine-needle aspiration (EUS-FNA) biopsy revealed the pancreatic tumor was compatible with metastasis of thymic AC. The patient underwent abdominal lymph node resection and distal pancreatectomy in June 2014. Because the lymph node on the ventral side of the branchiocephalic vein was judged as unresectable, 60-Gy radiation therapy was given in September 2014. Per pathological examination and immunohistochemistry, the final diagnosis was pancreatic metastasis of thymic AC. With several chemotherapy courses for recurrences, the patient has remained alive for eight years after the first surgery. Conclusion: AC metastasis to the pancreas is extremely rare. EUS-FNA is useful for diagnosis. Definitive determination of whether the treatment provides a survival benefit awaits further studies.
\end{abstract}

Keywords: thymic atypical carcinoid, pancreatic metastasis, distal pancreatectomy

Cite This Article: Masashi Inoue, Masahiro Tanemura, Toshimitsu Irei, Yuyo Maeda, Megumi Yamaguchi, Tatsuya Miyamoto, Shingo Seo, Toshihiro Misumi, Wataru Shimizu, Takahisa Suzuki, Takashi Onoe, Takeshi Sudo, Yosuke Shimizu, Takao Hinoi, and Hirotaka Tashiro, "A Case of Distal Pancreatectomy for Pancreatic Metastasis of Thymic Atypical Carcinoid.” American Journal of Medical Case Reports, vol. 5, no. 12 (2017): 285-288. doi: 10.12691/ajmcr-5-12-1.

\section{Introduction}

Neuroendocrine tumors are neoplasms that originate from the cells of the endocrine and nervous systems. Thymic neuroendocrine tumors(NECs), otherwise known as thymic carcinoid tumors, are uncommon primary thymic neoplasms. According to the 2015 World Health Organization Classification of Tumors of the Lung, Pleura, Thymus and Heart [1], thymic NECs are classified into four histological types: typical carcinoid (TC), atypical carcinoid (AC), small-cell neuroendocrine cell carcinoma (SCNEC), and large-cell neuroendocrine cell carcinoma (LCNEC). Most series have used the term 'carcinoid' to describe these tumors.

The majority of thymic NECs, which have poorly differentiated cells, have an aggressive biological behavior, that involves the bones, lungs, spleen, liver, and adrenal glands [2,3]. Even though ACs of the thymus are well-differentiated tumors, they have a high rate of recurrence and metastasis, with a 5-year overall survival rate of $56-77 \%$ [4] and a 10 -year overall survival rate of $30 \%$ [5]. Pancreatic metastasis of thymic AC is extremely infrequent.

Herein we report a case in which distal pancreatectomy was performed for pancreatic metastasis of thymic AC. In addition, to clarify associated clinical features, we present a summary of 3 cases of pancreatic metastasis of NEC and AC reported in the literatures.

\section{Case Report}

A 50-year-old man underwent thoracoscopic resection of a mediastinal tumor in June 2009. The diagnosis was thymic AC. He also underwent extended thymectomy in October 2011, thoracoscopic resection in October 2012, and tumor resection of the chest wall in March 2013 for recurrences. During follow up in April 2014, computed 
tomography (CT) revealed lymph node swelling on the ventral side of the brachiocephalic vein, around the left gastric vein, and on the left side of the celiac artery and suspected pancreatic tumor with low enhancement (Figure 1).
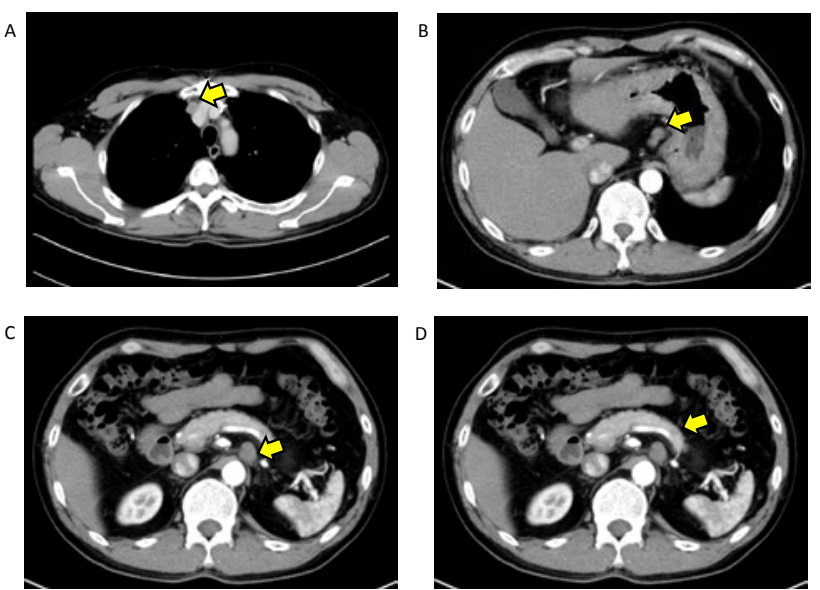

Figure 1. CT revealed lymph node swelling on the ventral side of the brachiocephalic vein(A), around the left gastric vein(B), and on the left side of the celiac artery(C), suspected pancreatic tumor with low enhancement (D)

A general physical examination and laboratory data were unremarkable. Fluorodexyglucose-positron emission tomography (FDG-PET) uptake was observed in each of the swollen lymph nodes, but no uptake was observed in pancreas (Figure 2).
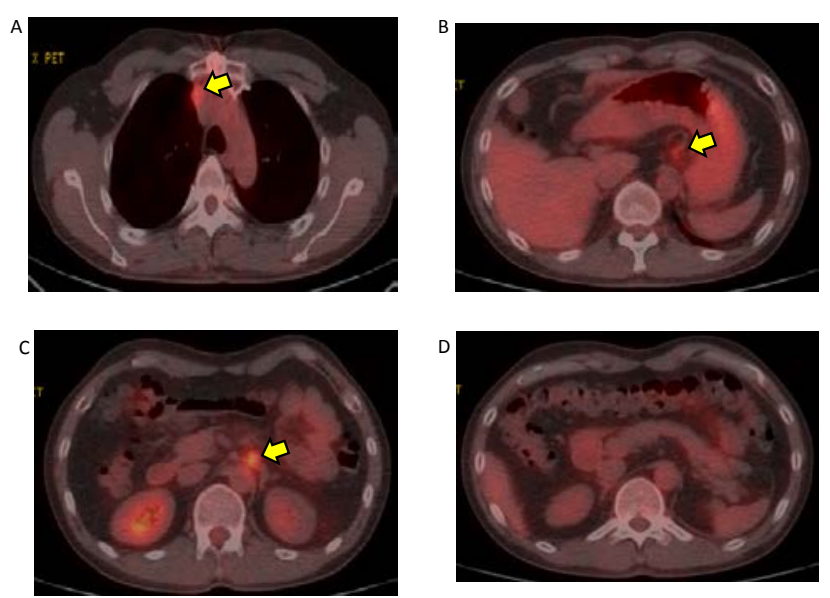

Figure 2. FDG-PET uptake was observed in each of the swollen lymph nodes(A,B,C) but no uptake was observed in pancreas(D)

Dynamic CT revealed a pancreatic tumor $10 \mathrm{~mm}$ in diameter with low enhancement (Figure 3).
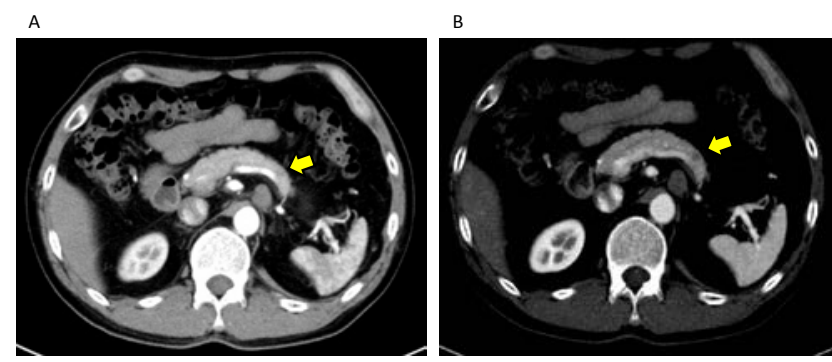

Figure 3. Dynamic CT revealed a pancreativ tumor $10 \mathrm{~mm}$ in diameter with low enhancement.
On endoscopic ultrasound (EUS), the tumor was appeared as a low-echoic lesion with distinct boundaries (Figure 4).

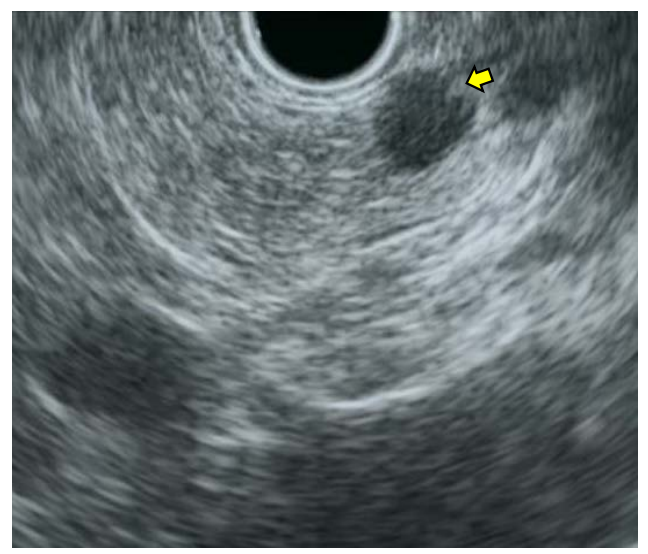

Figure 4. EUS revealed the tumor as a low-echoic lesion with distinct boundaries.

EUS-guided fine-needle aspiration (EUS-FNA) biopsy of the mass was performed, and on cytologic analysis, tumor cells with oval nuclei and acidophilic granular cytoplasm proliferating diffusely were observed. On Papanicolau staining, the nuclear chromatin appeared in a salt and pepper pattern (Figure 5).
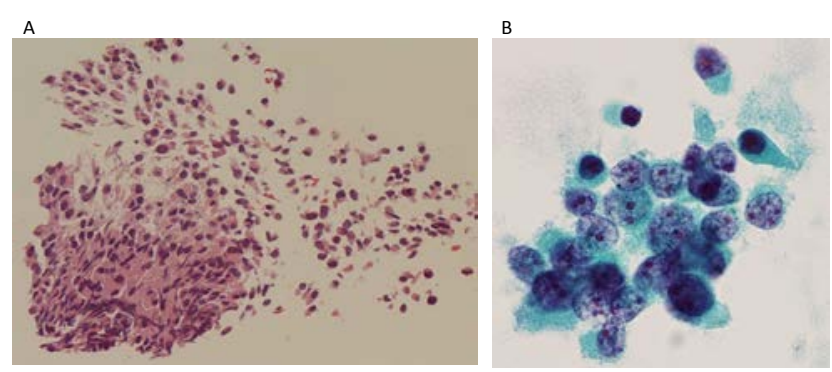

Figure 5. EUS-FNA biopsy of the mass was performed, and on cytologic analysis, tumor cells with oval nuclei and acidophilic granular cytoplasm proliferating diffusely were observed (A). On Papanicolau staining, the nuclear chromatin appeared in a salt and pepper pattern (B)

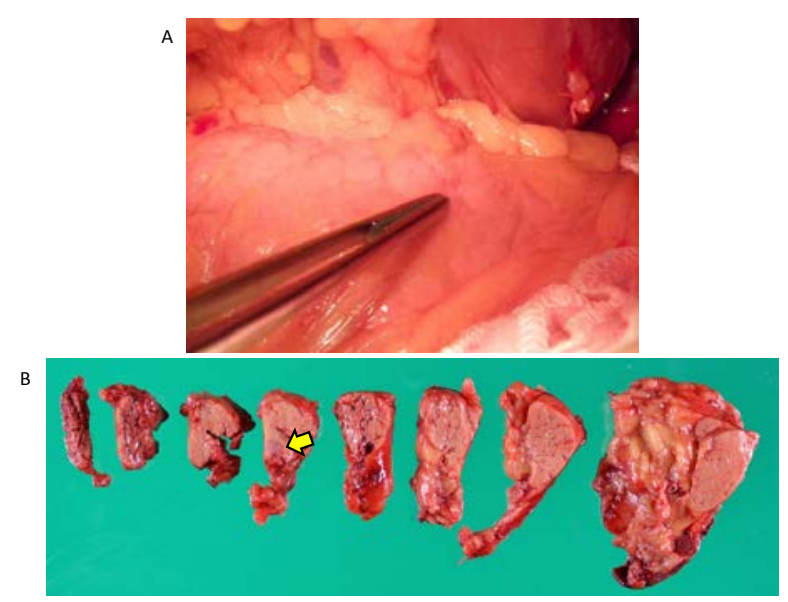

Figure 6. Macroscopically, the resected pancreatic tumor was elastic and soft, and measured $0.6 \times 0.3 \mathrm{~cm}$.

Thus, this pancreatic tumor was compatible with metastasis of thymic AC. The patient underwent abdominal lymph node resection and distal pancreatectomy in June 2014. Because the lymph node on the ventral side of 
the branchiocephalic vein was judged as unresectable, 60-Gy radiation therapy was given in September 2014. Macroscopically, the resected pancreatics tumor was elastic and soft, and measured $0.6 \times 0.3 \mathrm{~cm}$ (Figure 6).

Pathological examination revealed that the cytological morphology of the metastasis was identical to that of the originally resected thymic carcinoid. Immunohistochemistry revealed that the pancreatic tumor cells expressed chromogranin A and synaptophysin (Figure 7). The final diagnosis was pancreatic metastasis of thymic AC.
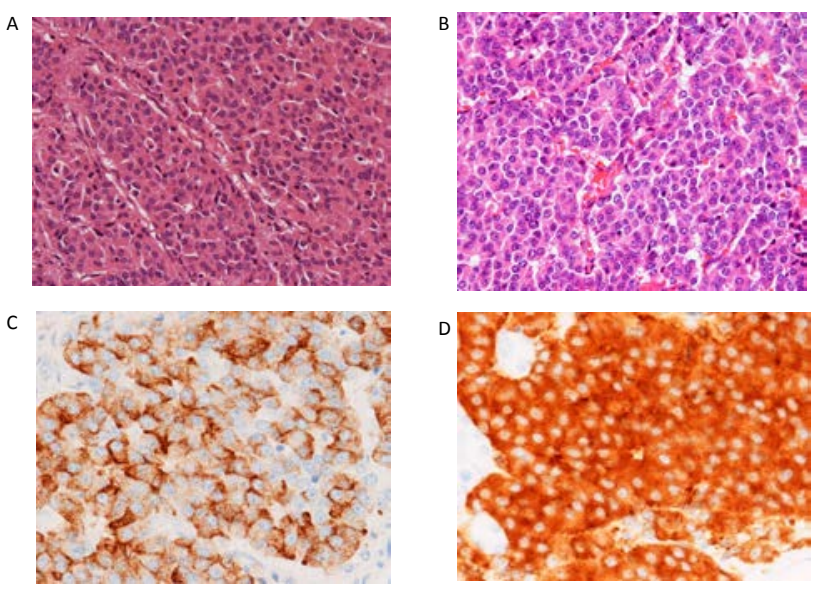

Figure 7. A) Thymic lesion. B) Pancreas lesion. Pathological examination revealed that the cytological morphology of the metastasis was identical to that of the originally resected thymic carcinoid. Immunohistochemistry revealed that the pancreatic tumor cells expressed chromogranin A (C) and synaptophysin (D).

In March 2013, CT revealed superior mediastinal metastasis. The patient received chemotherapy. The chemotherapy regimen was designed based on a therapeutic regimen for small-cell lung carcinoma, and included carboplatin (CBDCA) and irinotecan (CPT11). In May 2016, CT revealed abdominal para-aorta lymph node metastasis. Subsequent chemotherapy consisted of weekly nab-paclitaxel (nPTX) + CBDCA. The patient has remained alive for 8 years after the first surgery.

We comprehensively reviewed the literature describing pancreatic metastasis of thymic carcinoid or NEC. A PubMed search identified 3 cases, which are summarized in Table 1. Patient ages ranged from 36 to 65 years, and all patients were male. All patients underwent surgical resection of primary thymic tumor, and the interval to recurrence ranged from 1 year, 10 months to 5 years. Pancreatic tumor size ranged from 0.6 to $4.5 \mathrm{~cm}$, and 1 patient had multiple pancreatic tumors. One patient was diagnosed postoperatively, while the other two patients were diagnosed oreoperatively based on EUS-FNA. One patient underwent total pancreatectomy; the prognosis of this patient was described did not receive any postoperative therapy, and succumbed to the disease 9 months surgery.

\section{Discussion}

Thymic NEC is an extremely rare tumor that accounts for only $2-4 \%$ of all anterior mediastinal tumors [6,7]. Moreover, thymic AC represents an extremely rare type of thymic NEC, with an annual incidence of $\sim 0.18$ per $1,000,000$ peaple [8]. Thymic AC tends to have a more aggressive clinical course than TC. About 20\%-30\% of patients with thymic AC experience local recurrence or distant metastasis [9]. Pancreatic metastasis of thymic NEC, including AC, is extremely infrequent, and in the literature, to the best of our knowledge, only 3 cases have been previously reported $[10,11,12]$.

Thymic NECs are more common in men than in women, with the average age at diagnosis ranging from 42.0 to 56.5 years $[13,14,15]$. All 3 patients with pancreatic metastasis of thymic NEC and AC in the literature were male, with an average age at diagnosis of 53.7 years, which is consistent with that of all patients with thymic NEC. One patient evperienced symptoms of dull epigastric pain, while the other patients were asymptomatic.

In all 3patients, pancreatic tumors were detected by CT or magnetic resonance imaging (MRI). In 2 patients, pancreatic metastases were diagnosed by EUS-FNA, a modality that has been reported to be useful for diagnosing pancreatic masses, including NETs [16]. The accuracy of EUS-FNA has been reported to be $80 \%$ for diagnosing pNETs $[17,18]$. The concordance rate for grading pNETs between specimens obtained by EUS-FNA and surgery has been reported to be $87.5 \%$ [19]. Microscopically, thymic AC is well differentiated, with a mitotic index of $\geq 2 / 10$ HPF. On hematoxylin-eosin staining, of thymic NECs, dtumor cells are arranged in nested, trabecular, or pseudorosette patterns. Thymic NEC cells express neuroendocrine markers, including synaptophysin, chromogranin A, and neural cell adhesion molecule [13].

Lee et al. and Varytimiadis et al. reported thymic NEC cases diagnosed by EUS-FNA and immunohistochemistry. In the present case, the cytologic morphology was compatible with metastatic thymic carcinoid. In addition, immunohistochemical staining confirmed that the tumor contained neuroendocrine cells, so a diagnosis of metastatic thymic AC in the pancreas was made. As tumor cells were observed in lymphatic vessels, lymphogenous metastasis was suspected to be the mechanism of metastasis.

Table 1. Cases of pancreatic metastasis of thymic carcinoid or neuroendocrine carcinoma

\begin{tabular}{|c|c|c|c|c|c|c|c|c|c|}
\hline Author/year & Sex & Age & Thymic therapy & Interval & $\begin{array}{l}\text { Pancreas } \\
\text { tumor size }\end{array}$ & Diagnosis & Histology & Therapy & Prognosis \\
\hline LEE YT/ 2006 & 65 & M & Surgical removed & $2 \mathrm{Y}$ & $4 \mathrm{~cm}$ & EUS-FNA & NEC & Chemotherapy & - \\
\hline $\begin{array}{l}\text { Varytimiadis K/ } \\
2009\end{array}$ & 36 & M & Thymectomy & $5 Y$ & $\begin{array}{l}\text { Multiple } \\
\text { globular foci }\end{array}$ & EUS-FNA & NEC & Chemotherapy & - \\
\hline Yang DU/ 2016 & 60 & M & Resection & 1Y10M & $4.5 \times 4.1 \mathrm{~cm}$ & $\mathrm{CT}$ & NEC & $\begin{array}{c}\text { Total } \\
\text { pancreatectomy }\end{array}$ & 9 months died \\
\hline Our case/ 2017 & 51 & M & $\begin{array}{l}\text { Thoracoscopic } \\
\text { resection }\end{array}$ & $5 \mathrm{Y}$ & $0.6 \times 0.3 \mathrm{~cm}$ & EUS-FNA & $\begin{array}{l}\text { Atypical } \\
\text { carcinoid }\end{array}$ & $\begin{array}{c}\text { Distal } \\
\text { pancreatectomy }\end{array}$ & 3years alive \\
\hline
\end{tabular}


Surgery is critical for the treatment of patients with thymic AC, as the World Health Organization has suggested that this disease has a high malignant potential at all stages. For both unresectable or metastatic tumors, the National Comprehensive Cancer Network guidelines recommend chemotherapy with or without radiation therapy. However, due to their rarity, a clear role for radiation therapy or chemotherapy for metastatic lesions has not been established. In the present case, using the logic that resection of and radiation to each location would be more effective than systematic chemotherapy, abdominal lymph node resection and distal pancreatectomy were performed, and radiation therapy was directed to the area of mediastinal recurrence. Following systemic chemotherapy after the recurrence, the patient has remained alive for 8 years after the initial surgery.

Thymic AC metastasis to the pancreas may be very aggressive, but the present patient demonstrates that even in cases with systemic recurrent disease, multimodal treatment with radical intent, provides the possibility of long-term survival.

\section{Conclusion}

AC metastasis to the pancreas is extremely rare. EUSFNA is useful for diagnosis. Definitive determination of whether the treatment provides a survival benefit awaits further studies.

\section{References}

[1] Travis WD, Brambilla E, Burke AP, Marx A, Nicholson AG. WHO Classification of tumours of the Lung, Pleura, Thymus and Heart. Lyon: International Agency for Research on Cancer,2015

[2] Fukai I, Masaoka A, Fujii Y, Yamakawa Y, Yokoyama T, Murase $\mathrm{T}$, et al. Thymic neuroendocrine tumor (thymic carcinoid): A clinicopathologic study in 15 patients. Ann Thorac Surg 1999; 67: 208-211.

[3] de Montpréville VT, Macchiarini P, Dulmet E. Thymic neuroendocrine carcinoma (carcinoid): A clinicopathologic study of fourteen cases. J Thorac Cardiovasc Surg 1996; 111: 134-141.

[4] Han B, Sun JM, Ahn JS, Park K, Ahn MJ. Clinical outcomes of atypical carcinoid tumors of the lung and thymus: 7-year experience of a rare malignancy at single institute. Med Oncol. 2013; 30: 1-7.

[5] Ströbel P, Zettl A, Shilo K, et al. Tumor genetics and survival of thymic neuroendocrine neoplasms: a multi-institutional clinicopathologic study. Genes Chromosomes Cancer. 2014; 53: 738-749.

[6] Wick MR and Rosai J: Neuroendocrine neoplasms of the mediastinum. Semin Diagn Pathol.1991; 8: 35-51.

[7] Soga J, Yakuwa Y, Osaka M: Evaluation of 342 cases of mediastinal/thymic carcinoids collected from literature: A comparative study between typical carcinoids and atypical varieties. Ann Thorac Cardiovasc Surg.1999; 5: 285-292.

[8] Öberg K, Hellman P, Ferolla P, Papotti M. Neuroendocrine bronchial and thymic tumors: ESMO Clinical Practice Guidelines for diagnosis, treatment and follow-up. Ann Oncol. 2012; 7: 120-123.

[9] Moran CA, Suster S. Neuroendocrine carcinomas (carcinoid tumor) of the thymus. Am J Clin Pathol. 2000; 114: 100-110.

[10] Lee YT, Tse GM, Lai PB, Sung JJ. Metastatic thymic neuroendocrine carcinoma presenting as a pancreatic tumor. Endoscopy. 2006; 38: 58-59.

[11] Varytimiadis K, Kalaitzakis E, Salla C, Ghika E, Pandazopoulou A, Karoumpalis I. Pancreatic metastasis of thymic neuroendocrine carcinoma: Is there a role for endoscopic ultrasound? Report of a case and review of the literature. Pancreas. 2009; 38: 230-232.

[12] DU Y, Wang Y, Tang J, et al: Pancreatic metastasis resulting from thymic neuroendocrine carcinoma: A case report. Oncol Lett. 2016; 11: 1907-1910.

[13] Gaur P, Leary C, Yao JC. Thymic neuroendocrine tumors: a SEER database analysis of 160 patients. Ann Surg. 2010; 251: 11171121.

[14] Chaer R, Massad MG, Evans A, Snow NJ, Geha AS. Primary neuroendocrine tumors of the thymus. Ann Thorac Surg. 2002; 74: 1733-1740.

[15] Hage R, de la Rivière AB, Seldenrijk CA, van den Bosch JM. Update in pulmonary carcinoid tumors: a review article. Ann Surg Oncol. 2003; 10: 697-704.

[16] Larghi A, Iglesias-Garcia J, Poley JW, Monges G, Petrone MC, Rindi G, Abdulkader I, Arcidiacono PG, Costamagna G, Biermann $\mathrm{K}$, et al. Feasibility and yield of a novel 22-gauge histology EUS needle in patients with pancreatic masses: a multicenter prospective cohort study. Surg Endosc. 2013; 27: 3733-3738.

[17] Ardengh JC, de Paulo GA, Ferrari AP. EUS-guided FNA in the diagnosis of pancreatic neuroendocrine tumors before surgery. Gastrointest Endosc. 2004; 60: 378-384.

[18] Chatzipantelis P, Salla C, Konstantinou P, Karoumpalis I, Sakellariou S, Doumani I. Endoscopic ultrasound-guided fine-needle aspiration cytology of pancreatic neuroendocrine tumors: a study of 48 cases. Cancer. 2008; 114: 255-262.

[19] Mitsuru Sugimoto, Tadayuki Takagi, Takuto Hikichi, Rei Suzuki, Ko Watanabe, Jun Nakamura, et al. Efficacy of endoscopic ultrasonography-guided fine needle aspiration for pancreatic neuroendocrine tumor grading.World J Gastroenterol. 2015; 21: 8118-8124.

[20] Hage $R$, de la Rivière $A B$, Seldenrijk CA, van den Bosch JM. Update in pulmonary carcinoid tumors: a review article. Ann Surg Oncol. 2003; 10:697-704. 